\title{
A New Downlink Scheduling Algorithm Proposed for Real Time Traffic in LTE System
}

\author{
Mohammed Mahfoudi, Moulhime El Bekkali, Abdellah Najid, M. El Ghazi and Said Mazer
}

\begin{abstract}
The Third Generation Partnership Project (3GPP) has developed a new cellular standard based packet switching allowing high data rate, $100 \mathrm{Mbps}$ in Downlink and 50 Mbps in Uplink, and having the flexibility to be used in different bandwidths ranging from $1.4 \mathrm{MHz}$ up to $20 \mathrm{MHz}$, this standard is termed LTE (Long Term Evolution). Radio Resource Management (RRM) procedure is one of the key design roles for improving LTE system performance, Packet scheduling is one of the RRM mechanisms and it is responsible for radio resources allocation, However, Scheduling algorithms are not defined in 3GPP specifications. Therefore, it gets a track interests for researchers. In this paper we proposed a new LTE scheduling algorithm and we compared its performances with other well known algorithms such as Proportional Fairness (PF), Modified Largest Weighted Delay First (MLWDF), and Exponential Proportional Fairness (EXPPF) in downlink direction. The simulation results shows that the proposed scheduler satisfies the quality of service (QoS) requirements of the real-time traffic in terms of packet loss ratio (PLR), average throughput and packet delay. This paper also discusses the key issues of scheduling algorithms to be considered in future traffic requirements.
\end{abstract}

Keywords-LTE, QoS, Real Time Traffic, scheduling strategies.

\section{INTRODUCTION}

$\mathbf{T}$ HE QoS in LTE is influenced by a significant number of factors such as number of resources available, channel conditions and the type of services. LTE is based on Resource Block (RB) concept, which is a block of subcarriers with a number of consecutive subcarriers and a number of consecutive Orthogonal Frequency Division Multiplexing (OFDM) symbols in the frequency domain and the time domain respectively [1].

In Frequency Division Duplexing (FDD) operation mode and as shown in Figure 1, the LTE radio frame duration is 10 $\mathrm{ms}$, which is divided into $10 \mathrm{sub}$-frames of $1 \mathrm{~ms}$ each, which is also divided into two slots of $0.5 \mathrm{~ms}$ each. Each slot contains either six or seven OFDM symbols, depending on the Cyclic Prefix (CP) length [2].

A resource block is defined as a resource allocation unit where a pair of resource block is the minimum allocation unit, used by the scheduler while determining the allocations on a frame. For all the bandwidths the resource block size is the same. In frequency domain, one resource block is a constitution of 12 subcarriers length. In a $20 \mathrm{MHz}$ spectrum

M. Mahfoudi, M. EL Bekkali, S. Mazer, M. EL Ghazi are with the LTTI Laboratory, USMBA University, Morocco, Fes (email: mohammed. mahfoudi@usmba.ac.ma, moulhime.el.bekkali@gmail.com, mazersaid @gmail.com, elghazimo@hotmail.fr)

A. Najid is with the national institute of post and telecommunication, morocco, Rabat (email: najid@inpt.ac.ma).

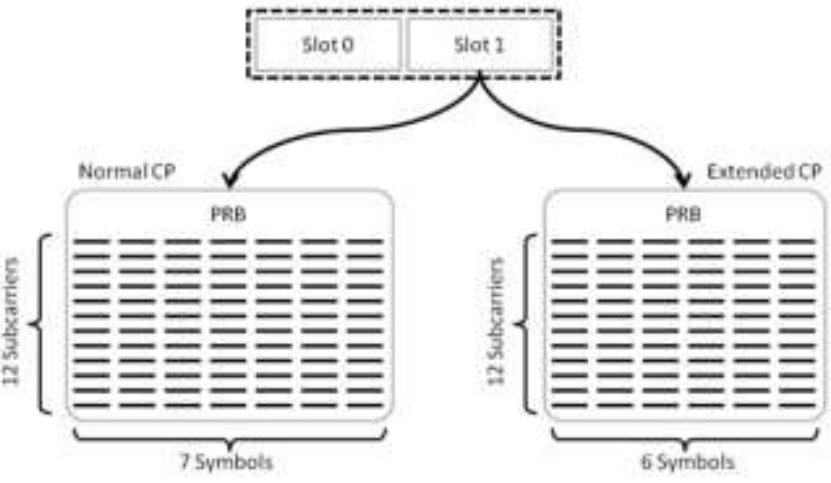

Fig. 1. LTE Frame structure [3]

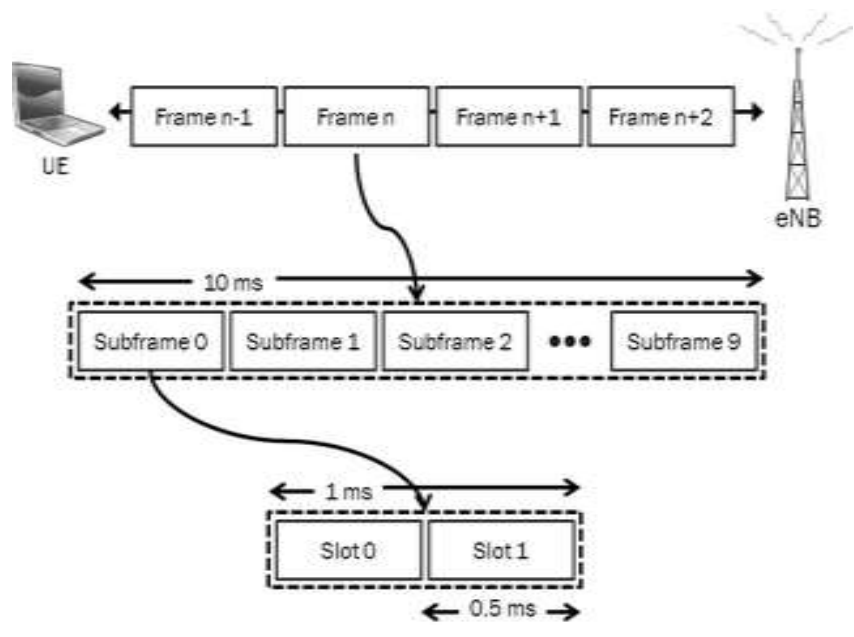

Fig. 2. Physical resource Block [3]

bandwidth, there are 1200 usable subcarriers and 100 Physical Resource Block (PRBs) [3].

The scheduling process is handled by the base station at the Medium Access Control (MAC) layer for LTE network. Uplink and downlink scheduling are separated in LTE and the scheduling decisions can be taken independently of each other [4]. The scheduler takes into account the channel conditions status labeled as channel quality indicator (CQI), which is updated regularly at each transmission time interval (TTI) [5].

\section{SCHEDUling STRATEGIES}

The LTE-SIM simulator models different uplink and downlink scheduling strategies in multi-cell/multi-user environments, it takes into account user mobility, radio 
resource optimization, frequency reuse techniques, the Adaptive Modulation, and Coding (AMC) module. It also includes other aspects that are relevant to the industrial and scientific communities [6].

3 GPP has been based on the redesign and simplification of the network architecture to an IP-based system, with significantly reduced transfer latency compared to the $3 \mathrm{G}$ architecture. The LTE Network must be operated on a separate wireless spectrum because of the incompatibility of its wireless interface with $2 \mathrm{G}$ and $3 \mathrm{G}$ networks In LTE network [7].

The radio protocols can be separated as shown in Figure 3 into control plane protocols which are used to carry the signaling between the User Equipment (UE) and user plane protocols which are used to carry user's data. The family of RRM algorithms at the LTE base station (eNodeB) exploits various functionalities from Layer 1 to Layer 3. Data is transferred between the MAC sublayers in the UE and eNodeB using transport blocks which are sent via the downlink and uplink shared transport channels [8].

At the Layer 1 the Channel Quality Indicator (CQI) manager processes the received CQI reports (downlink) and Sounding Reference Signals (SRSs) (uplink) from active users in the cell. For scheduling decisions, the eNodeB uses the received CQI report and SRS [9].

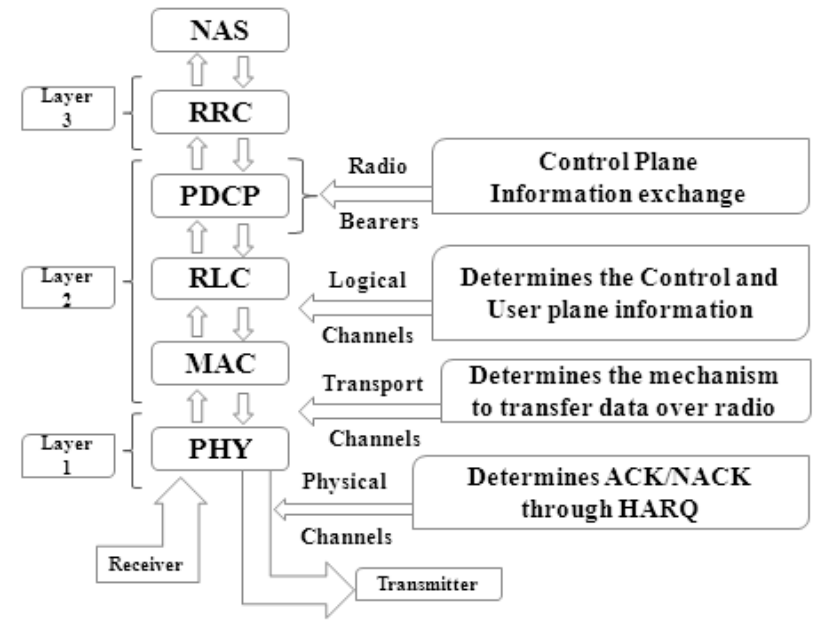

Fig. 3. LTE Protocols Stack

The main objectives of MAC sublayers within the eNodeB are to construct, send, receive and process transport blocks which contain a combination of user-plane data from one or more bearers. The MAC Scheduler runs the scheduling algorithms which determine how the downlink and uplink channels are used in the LTE air interface [9]. Resources are assigned to UEs enabling them to receive data via the downlink and transmit data via the uplink. The scheduler assigns these resources in such a way as to satisfy QoS requirements and optimize system performance.

In the downlink LTE system, a simplified packet scheduling model is shown in Figure 4.

The metric that can be allocated for each stream is calculated using different scheduler sequences. We assume that the metric assigned to stream $i$ on $j$-th sub-channel is defined by $w_{i, j}$, to obtain metric, schedulers need to know the average transmission rate $\bar{R}_{i}$ of flow $i$ and the available flow rate to the UE on the $j$-th sub-channel [10].

At each TTI, the estimate $\bar{R}_{i}$ is given by:

$$
\bar{R}_{i}(k)=0.8 \bar{R}_{i}(k-1)+0.2 R_{i}(k)
$$

Where $R_{i}(k)$ is the rate allocated to $i$-th flow during the $k$ th TTI and $\bar{R}_{\boldsymbol{i}}(k-1)$ is the average transmission data rate estimating at the $(k-1)$-th TTI.

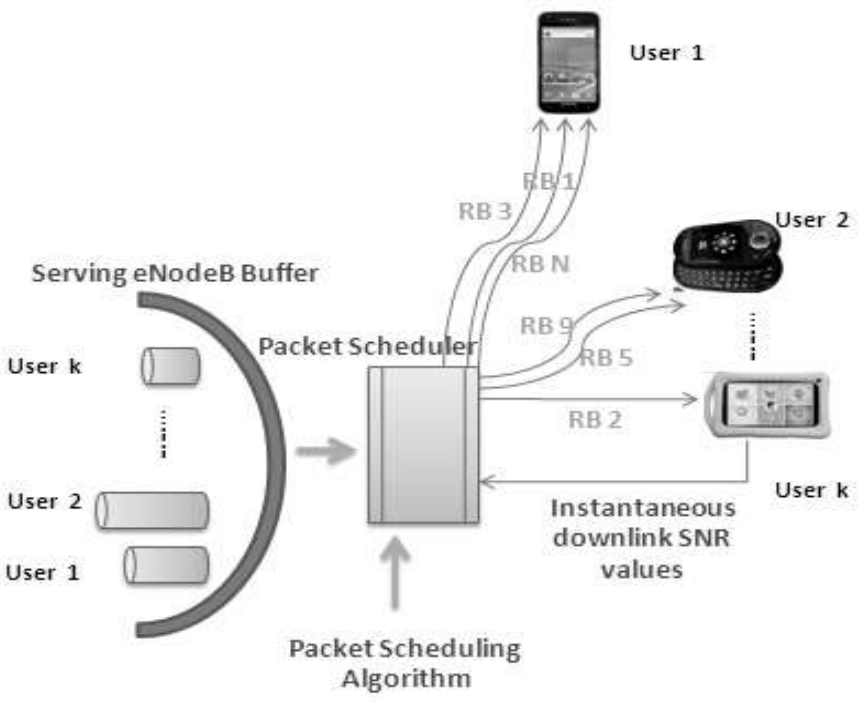

Fig. 4. Packet Scheduling Model in LTE System

In the following, a summary description of three different scheduling algorithms that are used in all simulation scenarios, these are: PF as well as EXP-PF and MLWDF.

\section{A. Proportional Fair $(P F)$}

The PF scheduler (Proportional Fair) assigns the radio resource considering the channel and the flow rate experienced by the user in the past quality. The goal is to maximize the total network throughput and ensure fairness between different data streams. For this scheduler, the metric east defines as the ratio between the instantaneous flow available for $i$-th flow and the medium flow was calculated at the moment $(k-1)$ [11].

$$
w_{i, j}=\frac{r_{i, j}}{\bar{R}_{i}}
$$

where $r_{i, j}$ is calculated by the AMC module, considering the CQI value on the $j$-th sub-channel which is sent by the UE who is intended for $i$-th flow.

\section{B. Exponential Proportional Fairness (EXP/PF)}

$\mathrm{EXP} / \mathrm{PF}$ algorithm configures the multimedia applications in a system of Adaptive Coding \& Modulation/Time Division Multiplexing (ACM/TDM) system. This type of algorithm can have both the real-time service user as well as non-real-time 
service and can enhance the priority of real-time flow with respect to non-real-time flow [12].

For real time flows, the metric is calculated by using the following equations:

$$
\mathrm{w}_{\mathrm{i}, \mathrm{j}}=\exp \left(\frac{\alpha_{\mathrm{i}} \mathrm{D}_{\mathrm{HOL}, \mathrm{i}}-\mathrm{X}}{1+\sqrt{\mathrm{X}}}\right) \frac{\mathrm{r}_{\mathrm{i}, \mathrm{j}}}{\bar{R}_{i}}
$$

Where $\mathrm{D}_{\mathrm{HOL}, \mathrm{i}}$ is the Head of line Delay, and $\mathrm{X}$ is given by:

$$
\mathrm{X}=\frac{1}{\mathrm{~N}_{\mathrm{rt}}} \sum_{i=\&}^{\mathrm{N}_{\mathrm{rt}}} \alpha \mathrm{D}_{\mathrm{i}} \mathrm{HOL}, \mathrm{i}
$$

With $\mathrm{N}_{\mathrm{rt}}$ is the number of active real time flows in downlink direction.

\section{Modified Largest Weighted Delay First (M-LWDF)}

The scheduler M-LWDF (Modified Largest Weighted Delay First) is designed to support multiple data users with diverse QoS requirements. For each real-time data streams, considering a time threshold $\tau$ packages, $\delta$ probability is defined as the maximum probability that the delay $D_{H O L, i}$, the first packet to be transmitted in the tail (Packet Head of Line) exceeds the threshold time [13].

To prioritize the real time flows having the highest time and the best conditions of propagation on the radio operator channel, the metric is defined in this scheduler by:

$$
w_{i, j}=\alpha_{i} D_{H O L, i} \frac{r_{i, j}}{\bar{R}_{i}}
$$

Where $r_{i, j}$ and $\bar{R}_{i}$ have the same signification as in the previous equation, and $\alpha \mathrm{i}$ is given by:

$$
\alpha_{i}=-\frac{\log \left(\delta_{i}\right)}{\tau_{i}}
$$

The network simulator LTE-Sim implements only FIFO (First In First Out) queues.

\section{THE PROPOSED SCHEDULING ALGORITHM}

In wireless communication systems, the channel conditions play an important role to maximize the throughput that is obtained by the user. We define $r_{i, j}$ as the current data rate that could be used by the $i$-th user on the $j$-th sub-channel at time t. This rate reflects the current condition of the user link. Thus users who have a better condition will get a higher priority to send their packages. So we can propose the metric as shown below:

$$
{ }_{i, j}=r_{i, j}
$$

However the users who don't have a better channel conditions will be punished. Therefore we need to consider the users who don't have a good channel conditions. Hence the necessity to consider the estimated average transmission data rate at the $(k-1)$-th Transmission Time Interval (TTI). So the new metric is the ratio between the current data rate that could be used by the $i$-th user on the $j$-th sub-channel and average transmission data rate at the moment $k-1$.

$$
w_{i, j}=\frac{r_{i, j}}{\bar{R}_{i}}
$$

Similar to MLWDF algorithm, for each real-time data streams, we define $\alpha i$ with considering $\tau$ as a time threshold packages and $\delta$ as the maximum probability that the delay $D_{H O L, i}$ exceeds the threshold time as follow[12]:

$$
\alpha_{i}=-\frac{\log \left(\delta_{i}\right)}{\tau_{i}}
$$

The second thing we need to consider is the delay, for the users who don't have better channel conditions, the probability that the Packet Head of Line delay exceeds the threshold time is bigger. Which can cause high packet loss ratio, high delay and low packet throughput.

To resolve this problematic, we considered the QoS information's sent to the eNodeB by the users especially the Head of line Delay $D_{H O L, i}$ for the $i$-th user the deadline delay $\tau_{\mathrm{i}}$, which are an important parameters that we must take on consideration, and to promote the users who don't have better channel conditions, we considered the following ratio that grow the metric value for users who have big Head of line Delay $D_{H O L, i}$, This term is explained as follow:

$$
\frac{\tau_{i}}{\left(\tau_{i}-D_{H O L, i}\right)}
$$

Normally the smaller the difference between the deadline delay and the time spend in queue $D_{H O L, i}$, the higher metric must be.

For the critical conditions this value must be more interesting, that's why we applied the exponential to the previous term. To give us the following term:

$$
\exp \left(\frac{\tau_{i}}{\left(\tau_{i}-D_{H O L, i}\right)}\right)
$$

Finally after combining all the cited parameters we obtained the proposed algorithm (LTTI_proposed) as defined below:

$$
w_{i, j}=\frac{\left(\alpha_{i}{ }^{*} r_{i, j}\right)}{\bar{R}_{i}} \exp \left(\frac{\tau_{i}}{\left(\tau_{i}-D_{H O L, i}\right)}\right)
$$

\section{Simulation AND PERFORMANCE EVAluATION}

LTE-SIM is an Open Source software Simulator, it was designed to make simulations for different scheduling strategies in uplink (UL) and downlink (DL) directions, and 
also it's used for multi-cell /multi-user environments considering the mobility of users, radio resources optimization, frequency reuse, adaptive modulation (AMC) and other significant aspects for industry and scientific community [14].

LTE-SIM allow network simulation according to the scenarios that the user decides, for example in our simulation we consider the case of a Single Cell with Interference, we used an environment with three cells with a radius of $1 \mathrm{Km}$ and in which a set of UE (selected in a range [5-20]) are uniformly in mobility $3 \mathrm{Km} / \mathrm{h}$ and are distributed in a cell, the two cells being a source of interference for the first one. UE moves into the cell following RANDOM_WALK Mobility Model [15]. Each user receives an H.264 Video stream, a VoIP stream, and BE flows modeled by Infinite Buffer.

The purpose of this simulation is to evaluate the performance of our new proposed scheduling algorithm in LTE Network, for this we compared its performances with the well known algorithms such as the PF, M-LWDF and EXP-PF schedulers [16], by measuring packet latency (delay), Packet Loss Ratio (PLR) and packets throughput. The simulation parameters are illustrated in the following Table.

TABLE I

SIMULATION PARAMETERS

\begin{tabular}{ll}
\hline Parameters & Value \\
Simulation duration & $100 \mathrm{~s}$ \\
Flows duration & $120 \mathrm{~s}$ \\
Frame structure & $F D D$ \\
Mobile speed & $3 \mathrm{Km} / \mathrm{h}$ \\
Radius & $1 \mathrm{~km}$ \\
Bandwidth & $10 \mathrm{MHz}$ \\
Slot duration & $0.5 \mathrm{~s}$ \\
Scheduling time (TTI duration) & $1 \mathrm{~ms}$ \\
Number of Resource Blocks (RBs) & 50 \\
Max delay & $0.1 \mathrm{~s}$ \\
Video bit-rate & $242 \mathrm{kbps}$ \\
VoIP bit-rate & $8.4 \mathrm{kbps}$ \\
Minimum number of users & 5 \\
Maximum number of users & 5 \\
Interval between users & 20 \\
\hline
\end{tabular}

\section{A. The Measurement of Packet Latency (Delay)}

Latency has a most noticeable influence on Network performance. Especially for conversational services, such as VoIP and Video Flows that require low latency [17]. Other services that benefit from low delay are gaming and applications with extensive handshaking, such as e-mail.

It is difficult to substantially improve latency without reducing the transmission time Interval (TTI)

\section{1) The VOIP Flows}

The Figure 5 demonstrates that the proposed algorithm presents a very low delay about $1.7 \mathrm{~ms}$ and its stable even the increase of users. The same behavior is observed for the PF and the EXP/PF algorithms. For the MLWDF the delay shows higher value comparing with the other algorithms by the increase of Users.

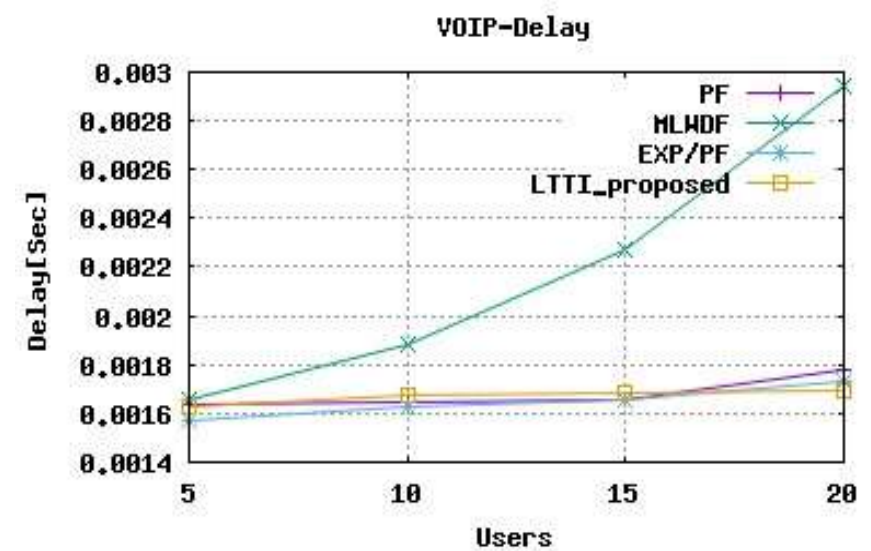

Fig. 5. The Delay for VOIP Flows

\section{2) For Video Flows}

As we can see in Figure 6, The Video delay is very low for our proposed scheduling algorithm, about [5-8]ms, even with the increment of users number, comparing with MLWDF and EXP/PF algorithms which present higher delay about [2050]ms for MLWDF and EXPPF. Unlike PF algorithm who presents dramatic increase until the value of $100 \mathrm{~ms}$ for 20 users.

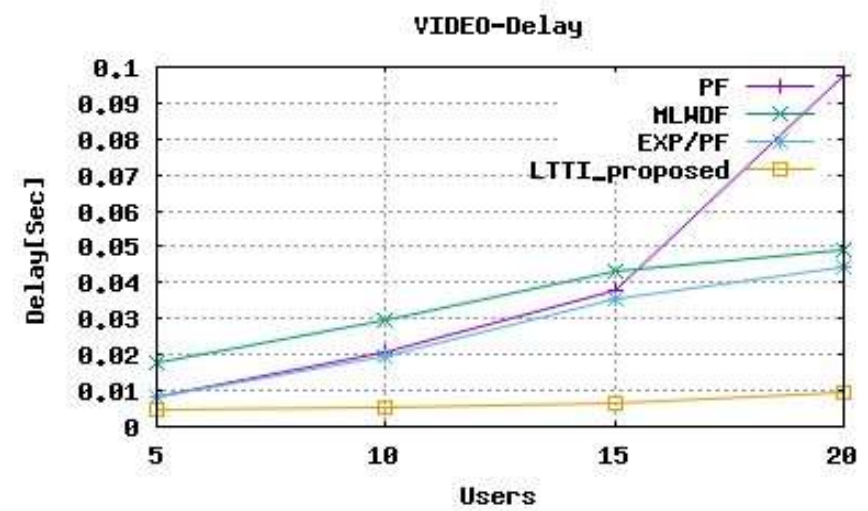

Fig. 6. The Delay for Video Flows.

\section{B. The Measurement of Packet Loss Ratio (PLR)}

The improvement of PLR estimation is a critical issue, because Packet Loss Ratio has a big effect on the network performance, especially when dealing with the real-time traffic such as VOIP and Video Flows [18].

\section{1) The VOIP Flows}

The Packet Loss Ratio has given in Figure 7, it shows that all the scheduling algorithms including our algorithm have a very negligible value of Packet Loss Ratio even the increase of user's number. 


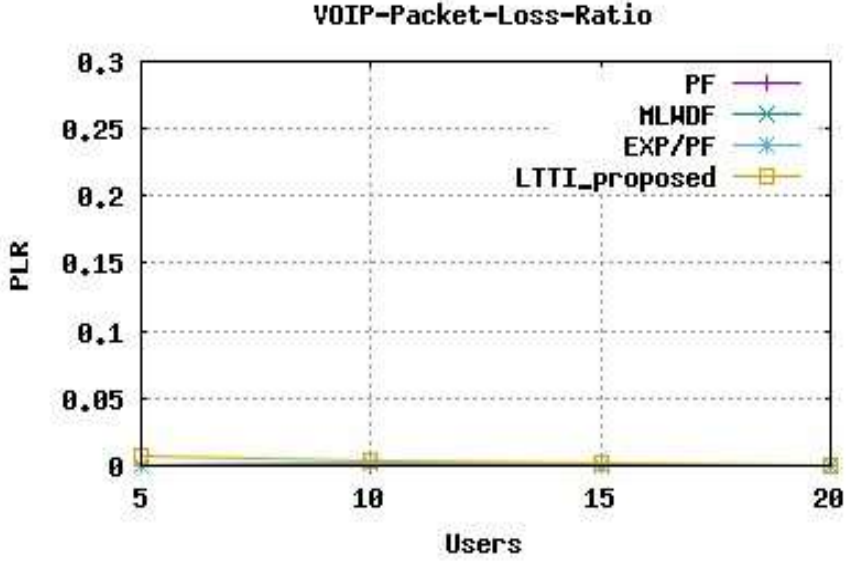

Fig. 7. The Packet Loss Ratio (PLR) for VoIP Flow

\section{2) For Video Flows}

The Packet Loss Ratio for video flows is given on Figure 8, it is noticed that the PLR given by our proposed algorithm is the lowest and is very negligible comparing with MLWDF, $\mathrm{EXP} / \mathrm{PF}$ and especially PF who shows a dramatic increase by the increasing of user's number.

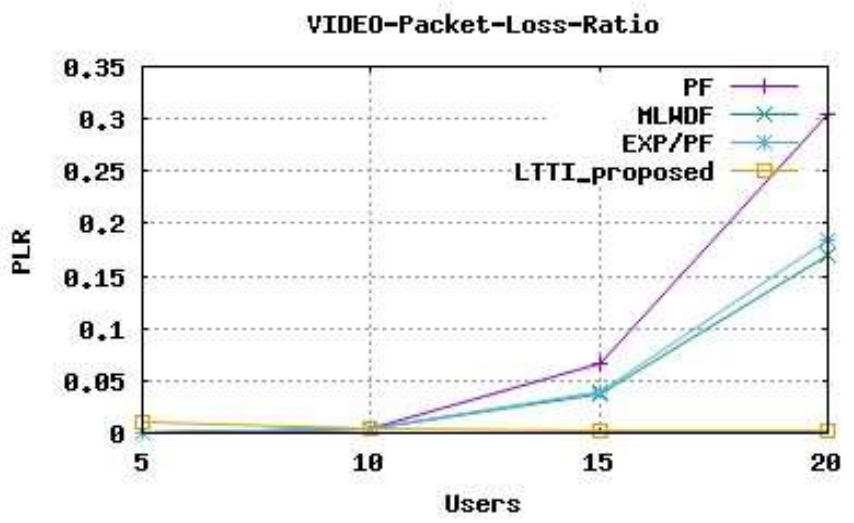

Fig. 8. The Packet Loss Ratio (PLR) for Video Flow

C. The Measurement of Packets Throughput

The Packets Throughput measurement is an important operation that allows identifying the average rate of successful message delivery over a communication channel [18].

\section{1) For VOIP Flows}

The Packet Throughput for VOIP Flows increases exponentially as the number of user's increases, and it's the same for all the Scheduling algorithms including the proposed one (Figure 9).

\section{2) For Video Flows}

The Video packet Throughput is given in Figure 10 shows that for the entire scheduling algorithms increases as Long as the number of users increases. Especially for our proposed scheduling algorithm which presents a high evolution of video packet Throughput.

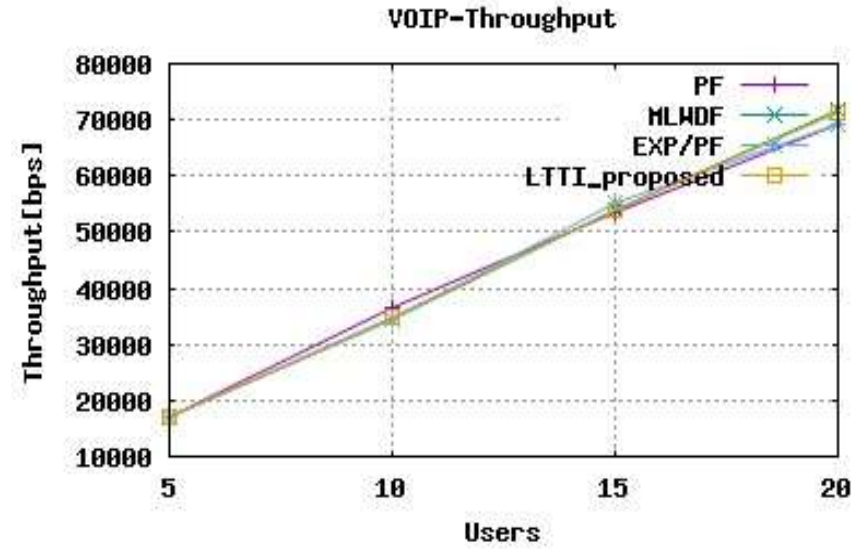

Fig. 9. The packets throughput for VOIP Flows.

VIDE0-Throughput

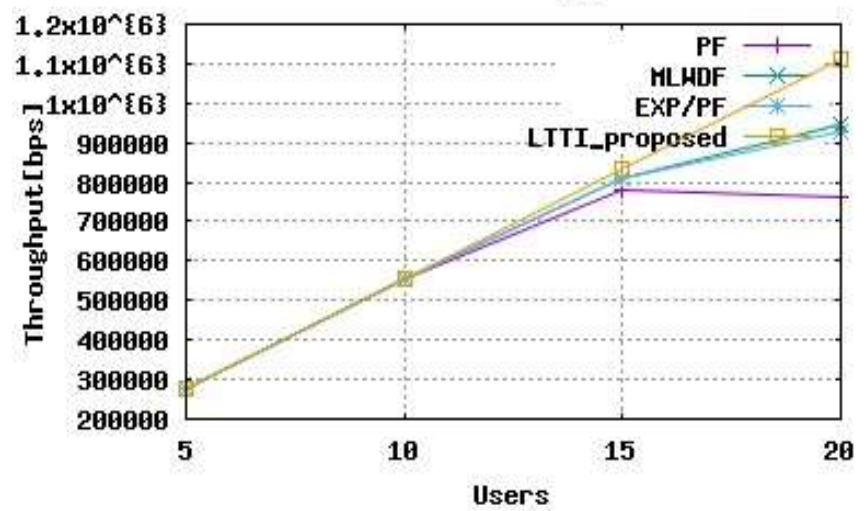

Fig. 10. The packets throughput for Video Flows.

\section{CONCLUSION}

In this paper, our work was focused on packet scheduling issues in the downlink of 3GPP LTE networks. We proposed a new algorithm and compared its performances with some well-known algorithms such as the proportional fair algorithm (PF), Exponential Proportional Fairness (EXP/PF) and the Modified Largest Weighted Delay First (MLWDF). Simulations have confirmed that our algorithm is more advanced and satisfies QoS requirements of Real Time services. It uses a metric based on CQI reporting and bearer priority for decision making.

Future work would aim to consider also the Non Real Time services to achieve the best overall system performance.

\section{REFERENCES}

[1] 3GPP TS 32.423 version 10.8.0, Digital cellular telecommunications system; LTE; Release10, April 2014.

[2] John Wiley \& Sons 'An Introduction to LTE: LTE, LTE-Advanced, SAE and 4G Mobile Communications, First Edition. ISBN: 9781119970385, Page (8-15) 2012.

[3] 3GPP, "Technical specifications group radio access network - physical channel and modulation (release 8)," 3GPP TS 36.211, 2009.

[4] T.Dikamba. "Downlink Scheduling in 3GPP Long Term Evolution “(LTE)”. MSc Thesis, TUDelft, March 2011. 
[5] Sun, Q., Tian, H., Dong, K., Zhou, R. and Zhang, P., "Packet Scheduling for Real-Time Traffic for Multiuser Downlink MIMO-OFDMA Systems," in IEEE Wireless Communications and Networking Conference, (2008), pp. 1849-1853.

[6] G Piro, LTE-Sim - the LTE simulator. [OnLine] Available: [http://telematics.poliba.it/LTE-Sim].

[7] 3GPP TS 23.002 V8.5.0, Network architecture (Release 8), June 2009.

[8] Technical White paper: LTE eNodeB MAC Scheduler Introduction, by Roke Manor Research, 2009.

[9] B. Sadiq, R. Madan and A. Sampath. "Downlink Scheduling for Multiclass Traffic in LTE". EURASIP Journal on Wirel. Comm. and Netw., vol. 2009 pp. 1-18, July. 2009.

[10] 3GPP TR 25.913 V8.0.0 LTE: Requirements for Evolved UTRA (EUTRA) and Evolved UTRAN (E-UTRAN) (Release 8), January 2009.

[11] F. Capozzi, G. Piro, L. Grieco, G. Boggia, and P. Camarda, "Downlink packet scheduling in lte cellular networks: Key design issues and a survey," Communications Surveys and Tutorials, IEEE, 2012.

[12] Rabie K. Almatarneh, Mohamed H. Ahmed, Octavia A. Dobre,' Performance analysis of Proportional Fair Scheduling in OFDMA Wireless Systems, "in proc. of 72nd IEEE Vehicular Tech. Conference, 6-9 Sept.,2010.
[13] R. Basukala, H. MohdRamli and K. Sandrasegaran. "Performance Analysis of EXP/PF and M-LWDF in Downlink 3GPP LTE System". IEEE F. Asian Himalayas Conf., pp. 1-5, Nov. 2009.

[14] G. Piro, L. Grieco, G. Boggia, F. Capozzi, and P. Camarda. "Simulating lte cellular systems: an open source framework". IEEE Trans. Veh. Technol., vol. 60, no. 2, pp. 498-513, Oct 2010 .

[15] D.Gillman," A Chernoff bound for random walks on expander graphs," Proc. 34th IEEE Symp. On Foundations of Computer Science (FOCS93), vol. SIAM J. Comp., Vol 27,No 4,1998.

[16] H. Ramli, R. Basukala, K. Sandrasegaran, and R. Patachaianand, "Performance of well known packet scheduling algorithms in the downlink 3GPP LTE system," in Proc. of IEEE Malaysia International Conf. on Comm., MICC, Kuala Lumpur, Malaysia, 2009, pp. 815-820.

[17] T. Blajić, D. Nogulić, M. Družijanić., Latency Improvements in $3 \mathrm{G}$ Long Term Evolution, Mobile Solutions. Ericsson Nikola Tesla d.d. (LATENCY),May 2007.

[18] E. Dahlman, H. Ekström, A. Furuskär, Y. Jading, J. Karlsson, M Lundevall, S. Parkvall, The 3G long-term evolution - radio interface concepts and performance evaluation, 63rd Vehicular Technology Conference, VTC 2006 - Spring, Vol. 1, pp. 137-141, IEEE, 2006. 